\title{
Detection rate for significant cancer at confirmatory biopsy in men enrolled in Active Surveillance protocol: 20 cores vs 30 cores vs MRI/TRUS fusion prostate biopsy
}

\author{
Pietro Pepe ${ }^{1}$, Sebastiano Cimino ${ }^{3}$, Antonio Garufi ${ }^{2}$, Giandomenico Priolo ${ }^{2}$, Giorgio Ivan Russo ${ }^{3}$, \\ Raimondo Giardina $^{3}$, Giulio Reale ${ }^{3}$, Michele Barbera ${ }^{1}$, Paolo Panella ${ }^{1}$, Michele Pennisi ${ }^{1}$, \\ Giuseppe Morgia ${ }^{3}$ \\ ${ }^{1}$ Urology and ${ }^{2}$ Imaging Unit, Cannizzaro Hospital, Catania, Italy; \\ ${ }^{3}$ Department of Urology, University of Catania, Italy.
}

\begin{abstract}
Summary Introduction: The detection rate for signifcant prostate cancer of extended vs saturation vs mMRI/TRUS fusion biopsy was prospectively evaluated in men enrolled in active surveillance (AS) protocol.

Mterials and methods: From May 2013 to September 201675 men aged 66 years (median) with very low risk PCa were enrolled in an AS protocol and elegible criteria were: life expectancy greater than 10 years, cT1C, PSA below $10 \mathrm{ng} / \mathrm{ml}$, PSA density $<0.20,2<$ unilateral positive biopsy cores, Gleason score (GS) equal to 6 , greatest percentage of cancer (GPC) in a core $<50 \%$. All patients underwent 3.0 Tesla pelvic mpMRI before confirmatory transperineal extended (20 cores) or saturation biopsy (SPBx; 30 cores) combined with mpMRI/TRUS fusion targeted biopsy (4 cores) of suspicious lesions (PI-RADS 3-5).

Results: 21/75 (28\%) patients were reclassified by SPBx based on upgraded GS $\geq 7$; mpMRI lesions PI-RADS 4-5 vs PI-RADS 3-5 diagnosed 9/21 (42.8\%) vs 16/21 (76.2\%) significant PCa with 2 false positives $(6.5 \%)$. The detection rate for significant PCa was equal to $76.2 \%$ (mpMRI/TRUS fusion biopsy) vs $81 \%$ (extended) vs $100 \%(S P B x)(p=0.001) ;$ mpMRI/TRUS targeted biopsy and extended biopsy missed 5/21 (23.8\%) and 4/21 (19\%) significant PCa which were found by SPBX $(p=0.001)$ being characterised by the presence of a single positive core of GS $\geq 7$ with GPC $<10 \%$.

Conclusions: Although mpMRI improve the diagnosis of clinically significant PCa, SPBX is provided of the best detection rate for PCa in men enrolled in AS protocols who underwent confirmatory biopsy.
\end{abstract}

KEY WORDS: Active Surveillance; Prostate cancer; Confirmatory prostate biopsy; MRI/TRUS fusion biopsy.

Submitted 15 Nivember 2016; Accepted 15 December 2016

\section{INTRODUCTION}

Active surveillance (AS) has become an alternative (1-3) to definitive treatment of low/very low risk prostate cancer (PCa), focusing on prevention of overtreatment (1) and strict monitoring over time of patients to establish potential risk reclassification. However, follow-up in the majority of AS protocols is still short, and prospective validation of criteria for selecting low-risk disease is still lack- ing (4). Recently, multi-parametric Magnetic Resonance Imaging (mpMRI) and mpMRI/TRUS fusion targeted biopsy have improved the accuracy of standard prostate biopsy schemes in the diagnosis of clinically significant PCa especially if located in the anterior prostate (5-7); therefore many authors suggest including mpMRI in AS follow up (8-13). However, the time of confirmatory biopsy has been established 6-12 months from initial diagnosis there are no data regarding the number of cores and the best procedure to diagnose exclusively clinically significant PCa.

In our study, the detection rate for PCa at confirmatory prostate biopsy has been prospectively evaluated performing mpMRI/TRUS fusion targeted biopsy vs extended or saturation prostate biopsy in men enrolled in a AS protocol study.

\section{Materials AND METHOdS}

From May 2013 to September 201675 men aged between 58 and 73 (median age 66) with very low risk PCa were enrolled in our AS protocol study. Presence of the following criteria defined eligibility: life expectancy greater than 10 years, clinical stage T1C, PSA below 10 ng/ml, PSA density (PSA-D) $<0.20,<2$ unilateral positive biopsy cores, Gleason score (GS) equal to 6, maximum core percentage of cancer (GPC) $<50 \%$.

All patients were requested to sign a written informed consent, and six months after PCa diagnosis underwent digital rectal examination, total PSA, PSA-D, PSA doubling time measurement and pelvic mpMRI 3.0 Tesla evaluation before confirmatory trans-perineal prostate saturation biopsy (SPBx); the procedure was performed with the use of a GE Logiq P6 ecograph (General Electric; Milwaukee, WI) supplied with a bi-planar trans-rectal probe (5-7.5 $\mathrm{MHz}$ ) using a tru-cut 18 gauge needle (Bard; Covington, GA) under sedation and antibiotic prophylaxis (14). All mpMRI examinations were performed using a 3.0 Tesla scanner, (ACHIEVA 3T; Philips Healthcare Best, the Netherlands) equipped with surface 16 channels phasedarray coil placed around the pelvic area with the patient in the supine position; multi-planar turbo spin-echo T2- 
weighted (T2W), axial diffusion weighted imaging (DWI), axial dynamic contrast enhanced (DCE) and spectroscopy were performed for each patient. The mpMRI lesions characterized by a PI-RADS (Prostate Imaging-Reporting and Data System) score of 4 and 5 were considered highly suspicious for cancer $(6,7)$; two radiologists (AF, GP) blinded to pre-imaging clinical parameters evaluated the MRI data separately and independently.

In the presence of mpMRI lesions suggestive of cancer (PI-RADS 3-5), targeted MRI/TRUS fusion guided-biopsies were added to standard SPBx using a GE Logiq E9 (General Electric; Milwaukee, WI) or Hitachi Arietta 70 ecograph (Hitachi Medico, Chiba, Japan) supplied with a end-fire (Figure 1) or biplanar transrectal (Figure 2) probe, respectively. Risk reclassification at repeat biopsy triggered the recommendation for active treatment and defined as over 3 or more than $10 \%$ of positive cores, GS $>6$, GPC > 50\%; patients being reclassified underwent definitive treatment (radical prostatectomy or external radiotherapy).

We evaluated the detection rate for clinically significant PCa performing extended biopsy (20 cores: 16 in the periphery and 4 in the anterior zone) vs saturation biop-

\section{Figure 1.}

Multiparametric transrectal MRI/TRUS fusion targeted prostate biopsy of a suspicious lesion located in the left (target 1) of the gland (PI-RADS 3).

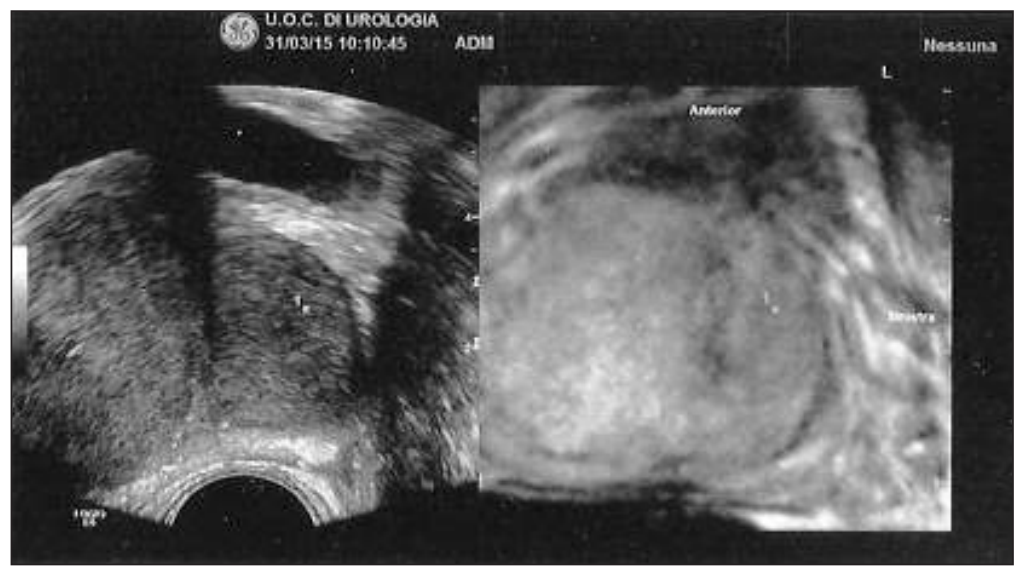

Figure 2.

Multiparametric transperineal MRI/TRUS fusion targeted prostate biopsy of a suspicious lesion located in the anterior prostate (target lesion: PI-RADS 4).

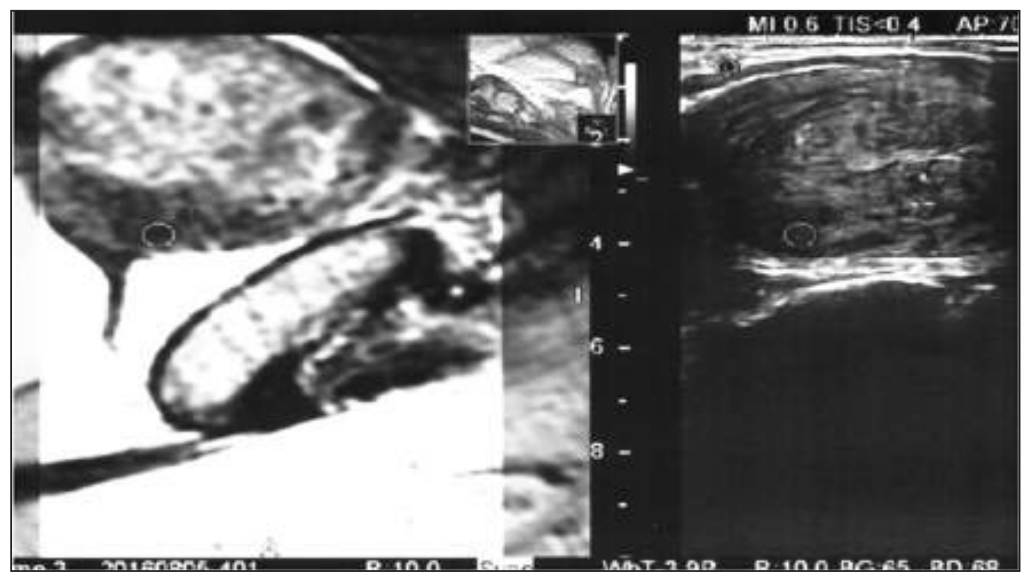

sy (30 cores: 24 in the periphery and 6 in the anterior zone) vs mpMRI/TRUS fusion guided-biopsies (4 targeted cores of the suspicious lesions with PI-RADS 3-5). Probability level of $\mathrm{p}<0.05$ was considered statistically significant

\section{RESULTS}

The clinical parameters of the 75 patients enrolled in the AS protocol are listed in the Table 1. 21/75 (28\%) patients had unfavourable repeat SPBx and were reclassified based on upgraded GS ( 15 cases GS $=3+4 ; 4$ cases $\mathrm{GS}=4+3 ; 2$ cases $\mathrm{GS}=4+4)$ and number of positive cores (range: $3-5$ positive cores; $50 \%$ of the cases). In detail, 8 (38\%) PCa were located only in the anterior zone of the gland, 8 (38\%) in the periphery zone and 5 (24\%) in both zones. Of the remaining 54 (72\%) patients, 33 were found to have very low-risk PCa and in 21 cancer was absent; PCa was located in the periphery in 22 cases and in the anterior zone in 11 cases. A total of 124 (mpMRI/TRUS fusion targeted biopsy) vs 2250 (SPBx) vs 1500 (extended biopsy) cores were performed and the detection rate of $\mathrm{PCa}$ for single core was equal to $22.5 \%$ vs $5 \%$ vs $6.8 \%$, respectively.

No-one suffered significant complication from standard biopsy (extended or SPBx) or mpMRI/TRUS fusion targeted biopsy requiring admission to Hospital. Multiparametric pMRI was suspicious (PIRADS $3-5)$ in 31 of 75 cases (41.3\%); in detail, mpMRI showed a lesion with PI-RADS 4-5 in all the patients with GS $4+4$ (2/2 cases) and GS $4+3$ (4/4 cases), in 3/15 (20\%) men with GS $3+4$ and in 2 patients who were not reclassified. In addition, mpMRI found lesions with PI-RADS 3 in the 7/15 (46.6\%) patients with GS $3+4$ and in the remaining 13 (17.4\%) cases who were not reclassified. High level of concordance in the diagnosis of PI-RADS 3-5 between the two radiologists was found (Cohen's Kappa 0.85). Diagnostic accuracy, sensitivity, specificity, positive and negative predictive value (NPV) of mpMRI in diagnosing significant PCa in the presenc of PI-RADS 3-5 vs PI-RADS 4-5 were: 83.4 vs $84.3 \%, 76.5$ vs $42.8 \%, 84.3$ vs $96.4 \%, 51$ vs $81 \%, 96.4$ vs $100 \%$, respectively.

Twelve upgraded patients underwent radical prostatectomy (6 open surgery, 2 laparoscopic and 4 robotic prostatectomies); in every case, definitive pathology report found organ confined PCa of GS 7 with negative surgical margins and nodes (pT2cNO); in addition, 4 men with negative mpMRI who chose radical prostatectomy (through anxiety), were found to have clinically insignificant PCa (cancer volume $<0.5 \mathrm{ml}$ and a $6 \mathrm{GS})(15)$.

Summing up, mpMRI lesions PI-RADS 4-5 vs PI-RADS 3-5 diagnosed 9/21 (42.8\%) vs 16/21 (76.2\%) significant PCa (8) charac- 


\section{Table 1.}

Clinical parameters of the 75 men enrolled in the Active Surveillance protocol who underwent confirmatory prostate biopsy.

\begin{tabular}{|lc|}
\hline Median PSA (range) & $6.5 \mathrm{ng} / \mathrm{ml}(3.7-10 \mathrm{ng} / \mathrm{ml})$ \\
\hline Median PSA-D (range) & $0.15(0.09-0.20)$ \\
\hline DRE & negative \\
\hline Gleason score & $6(3+3)$ \\
\hline GPC (range) & $20 \%(5-50 \%)$ \\
\hline $\begin{array}{l}\text { Prostate weight (range) } \\
\text { Median number of positive cores }\end{array}$ & 48 grams (30-115 grams) \\
\hline
\end{tabular}

terised by GS $\geq 7$ (100\% of the cases) and a number of positive core $>3$ ( $50 \%$ of the cases) with 2 false positives (6.5\%). The detection rate for significant PCa was equal to $76.2 \%$ (mpMRI/TRUS fusion biopsy) vs 81\% (16 p) (extended) vs 100\% (SPBx 21) ( $\mathrm{p}=0.001$ ); in detail, mpMRI/TRUS targeted biopsy and extended biopsy missed 5/21 (23.8\%) vs 4/21 (19\%) significant PCa which were found by SPBx being characterised by the presence of a single positive core of GS $\geq 7$ with GPC $<10 \%$.

\section{Discussion}

There are many published active surveillance series, varying in size of population and duration of follow-up (1-4) those have mostly restricted this approach to favourable-risk patients; there is considerable variation regarding patient selection, follow-up policies and when active treatment should be offered. Although biological markers appear promising as does genomics on the tissue sample itself, follow up in AS should be based on serial PSA measurements, PSA kinetics (PSA doubling time), clinical examination and, in particular, repeat prostate biopsy; in this respect, the percentage of patients reclassified at confirmatory prostate biopsy reported in the Prostate Cancer Research International Active Surveillance (PRIAS) study (1) is equal to $28 \%$ (415/2494 cases). Although the optimal number of cores (extended vs saturation biopsy) and approach of prostate biopsy (trans-rectal vs trans-perineal) has not been established (16), the criteria of reclassification include upgrading ( $G S \geq 7)$ and modification of biopsy quantitative histology (number of positive cores, GPC > 50\%). SPBx in comparison with extended biopsy (17) demonstrated more accurate assessment of the extent and grade of disease in men enrolled in AS protocol; in addition, the trans-perineal free hand or template SPBx increases progression to treatment in AS (18) improving the detection rate of PCa located solely in the anterior zone of the gland (about $10 \%$ of the cases) $(19,20)$.

In the last years, mpMRI and mpMRI/TRUS fusion targeted biopsy have a good degree of accuracy in diagnosing clinically significant PCa secondary to the high sensitivity for lesion upgrading, especially when the cancer is located in the anterior prostate $(6,7)$; mpMRI targeted biopsy allows to reclassify about $10 \%$ of patients eligible for AS in comparison with standard trans-rectal biopsy $(21,22)$. In addition, if confirmed by larger studies,
mpMRI could be useful to better define those having very low risk PCa allowing for a greater interval of time for prostate biopsy re-evaluation (23) and reducing the risk of clinical complications secondary to repeat biopsy (24). On the other hand, false negative rate of mpMRI in diagnosing significant PCa is equal to $15-30 \%$ of the cases especially in the presence of low volume of PCa with GS $\geq 7$ (25); in this respect, a combination of systematic and MRI/TRUS fusion targeted cores increase detection on significant PCa (26-28).

In our series, we found mpMRI to have a $83.4 \%$ (PIRADS 3-5) diagnostic accuracy rate with a $94.6 \%$ NPV rate in predicting the presence of clinically significant PCa; mpMRI/TRUS targeted biopsy and extended biopsy missed 5/21 (23.8\%) and 4/21 (19\%) significant PCa which were found by SPBx $(p=0.001)$ being characterised by the presence of a single positive core of GS $\geq$ 7 with GPC $<10 \%$.

These data suggest that mpMRI/TRUS fusion biopsies alone could miss small but significant PCa because mpMRI accuracy significantly correlates with the diameter of the suspicious lesions $(7,29)$; moreover, in a selected population of men with very low-risk PCa in AS with an expected negative mpMRI, the suspicious lesions with PI-RADS 3 should undergo targeted biopsy to improve the reclassification of the patients. In definitive, SPBx detect the highest percentage of PCa allowing to better define the best therapeutic clinical strategy for each patient.

Limitations and considerations of the present study need mention. Firstly, a greater number of patients need to be examined; secondly, we do not know the true diagnostic accuracy of mpMRI and biopsy procedure in PCa diagnosis because the detection rate for cancer was compared only in 16/75 (21.3\%) cases with definitive specimen. Finally, we cannot establish if the two mpMRI false positives were possibly re-assignable to false-negative repeat prostate biopsy procedures (30).

In conclusion, although mpMRI improve the diagnosis of clinically significant PCa, SPBx is provided of the best detection rate for $\mathrm{PCa}$ in men enrolled in AS protocols who underwent confirmatory biopsy.

\section{References}

1. Schröder FH, Hugosson J, Roobol MJ, et al: Prostate-cancer mortality at 11 years of follow-up. N Engl J Med. 2012; 366:981-990.

2. Bokhorst LP, Zappa M, Carlsson SV, et al. Correlation between stage shift and differences in mortality in the European Randomised study of Screening for Prostate Cancer (ERSPC). BJU Int. 2016; 118:677-680.

3. Richard PO, Alibhai SM, Panzarella T, et al. The uptake of active surveillance for the management of prostate cancer: A populationbased analysis. Can Urol Assoc J. 2016; 10:E342-E346.

4. Kim TH, Jeon HG, Choo SH, et al. Pathological upgrading and upstaging of patients eligible for active surveillance according to currently used protocols. Int J Urol . 2014; 21:377-381.

5. Pepe P, Garufi A, Priolo G, Pennisi M. Can 3 Tesla pelvic phasearray MRI avoid unnecessary repeat prostate biopsy in patients with PSA below 10 ng/ml? Clinical Genitourinary Cancer. 2015; 13:e27-30. 
6. Pepe P, Garufi A, Priolo G, et al. Prostate cancer detedtion at repeat biopsy: can pelvic phased-array multiparametric MRI replace saturation biopsy? Anticancer Research. 2013; 33:1195-1199.

7. Pepe P, Garufi A, Priolo G, Pennisi M. Transperineal versus transrectal MRI/TRUS fusion targeted biopsy: detection rate of clinically significant prostate cancer. Clin Genitourin Cancer. 2016 Jul 21. pii: S1558-7673(16)30208-7. doi: 10.1016/j.clgc.2016.07.007. [Epub ahead of print]

8. Kuru TH, Roethke MC, Seidenader J, et al. Critical evaluation of magnetic resonance imaging targeted, transrectal ultrasound guided transperineal fusion biopsy for detection of prostate cancer. J Urol. 2013; 190:1380-1386.

9. Fascelli M, George AK, Frye T, et al. The role of MRI in active surveillance for prostate cancer. Curr Urol Rep 2015; 16:42.

10. Guo R, Cai L, Fan Y, et al. Magnetic resonance imaging on disease reclassification among active surveillance candidates wiht lowrisk prostate cancer; a diagnostic metanalysis. Prostate Cancer Prostatic Dis. 2015; 18:221-8.

11. Schoots IG, Petrides N, Giganti F, et al. Magnetic resonance imaging in active surveillance of prostate cancer: a systematic review. Eur Urol. 2015; 67:627-636.

12. Perera M, Katelaris N, Murphy D, et al. PI-RADS 4 or more: Active Surveillance no more. BJU Int. 2016. doi: 10.1111/bju.13562. [Epub ahead of print]

13. Vos LJ, Janoski M, Wachowicz K, et al. Role of serial multiparametric magnetic resonance imaging in prostate cancer active surveillance. World J Radiol. 2016; 8:410-418.

14. Pepe P, Aragona F. Saturation prostate needle biopsy and prostate cancer detection at initial and repeat evaluation. Urology. 2007; 70:1131-1135.

15. Epstein J, Walsh P, Carmichael M. Pathological and clinical findings to predict tumor extent of non palpable (stage T1c) prostate cancer. JAMA. 1994; 271:368-374.

16. Klein EA, Cooperberg MR, Magi-Galluzzi C, et al. A 17-gene assay to predict prostate cancer aggressiveness in the context of Gleason grade heterogeneity, tumor multifocality, and biopsy undersampling. Eur Urol. 2014; 66:550-560.

17. Chung PH, Darwish OM, Roehrborn CG, et al. Histologic upgrading in patients eligible for active surveillance on saturation biopsy. Can J Urol. 2015; 22:7656-7660.

18. Abouassaly R, Lane BR, Jones JS. Staging saturation biopsy in patients with prostate cancer on active surveillance protocol. Urology. 2008; 71:573-577.

19. Thompson JE, Hayen A, Landau A, et al. Medium.term oncological outcomes for estende vs saturation biopsy and transrectal vs tranperineal biopsy in active surveillance for prostate cancer. BJU Int. 2015; 115:884-891.

20. Pepe P, Dibenedetto G, Pennisi $M$, et al. Detection rate of anterior prostate cancer in 226 patients submitted to initial and repeat transperineal biopsy. Urol Int. 2014; 93:189-192.

21. Pham KN, Porter CR, Odem-Davis K, et al. Transperineal template guided prostate biopsy selects candidates for active surveillance: how many cores are enough? J Urol. 2015; 194:674-679.

22. Ouzzane A, Renard-Penna R, Marliere F, et al. Magnetic resonance imaging targeted biopsy improves selection of patients considered for active surveillance for clinically low risk prostate cancer based on systematic biopsies. J Urol. 2015; 194:350-6.

23. Kamrava M, Kishan AU, Margolis DJ, et al. Multiparametric magnetic resonance imaging for prostate cancer improves Gleason score assessment in favorable risk prostate cancer. Pract Radiat Oncol. 2015; 5:411-6

24. Satasivam P, Poon BY, Ehdaie B, et al. Can confirmatory biopsy be omitted in prostate cancer active surveillance patients with favorable diagnostic features? J Urol. 2016; 195:74-9

25. Pepe P, Aragona F. Morbidity following transperineal prostate biopsy in 3,000 parients submitted to 12 vs 18 vs more than 24 needle cores. Urology. 2013; 81:1142-1146.

26. Pepe P, Garufi A, Priolo G, Pennisi M. Can MRI/TRUS fusion targeted biopsy replace saturation prostate biopsy in the re-evaluation of men in active surveillance? World J Urol. 2016; 34:12491253

27. Radtke JP, Kuru TH, Boxler S, et al. Comparative analysis of transperineal template saturation prostate biopsy versus magnetic resonance imaging targeted biopsy with magnetic resonance imaging-ultrasound fusion guidance. J Urol. 2015; 193:87-94.

28. Tran M, Thompson J, Böhm M, et al. Combination of multiparametric MRI and transperineal template-guided mapping biopsy of the prostate to identify candidates for hemi-ablative focal therapy. BJU Int. 2016; 117:48-54.

29. Ma TM, Tosoian JJ, Schaeffer EM, et al. The role of multiparametric magnetic resonance imaging/ultrasound fusion biopsy in active surveillance.Eur Urol. 2016 May 25. pii: S03022838(16)30185-3. doi: 10.1016/j.eururo.2016.05.021. [Ерub ahead of print]

30. Wegelin O, van Melick HH, Hooft L, et al. Comparing three different techniques for magnetic resonance imaging-targeted prostate biopsies: a systematic review of in-bore versus magnetic resonance imaging-transrectal ultrasound fusion versus cognitive registration. Is there a preferred technique? Eur Urol. 2016. pii: S03022838(16)30446-8. doi: 10.1016/j.eururo.2016.07.041. [Epub ahead of print]

\section{Correspondence}

Pietro Pepe, MD (Corresponding Author)

piepepe@hotmail.com

Urology Unit, Cannizzaro Hospital

Via Messina 829, Catania, Italy

Sebastiano Cimino, MD

Giorgio Ivan Russo, MD

Raimondo Giardina, MD

Giulio Reale, MD

Michele Barbera, MD

Paolo Panella, MD

Michele Pennisi, MD

Giuseppe Morgia, MD

Department of Urology, University of Catania, Catania, Italy

Antonio Garufi, MD

Giandomenico Priolo, MD

Imaging Unit, Cannizzaro Hospital, Catania, Italy 